\title{
Vicarious trial-and-error is associated with the use of place-strategies in human virtual navigation
}

\author{
Neil Schmitzer-Torbert \\ Wabash College
}

\begin{abstract}
Author Note
Neil Schmitzer-Torbert (iD) https://orcid.org/0000-0001-6186-7853
\end{abstract}

I have no known financial conflict of interest to disclose.

A preprint of this manuscript is available at https://psyarxiv.com/f8rhn/.

Correspondence concerning this article should be addressed to Neil Schmitzer-Torbert, Department of Psychology, Wabash College, 301 W. Wabash Ave, Crawfordsville, IN 47933. Email: torbertn@wabash.edu. 


\begin{abstract}
Studies of decision-making in rodents have demonstrated that vicarious trial-and-error (VTE) is an important behavioral index of deliberation, when animals search through and evaluate the available options before making a decision. In rodents, VTE is enhanced during the use of hippocampally-dependent place strategies, which may represent a type of model-based behavior. While some evidence exists for VTE-like behaviors in humans during navigation, it is unknown if VTE in humans is specifically associated place-strategies, as would be predicted for modelbased behaviors. To address this gap, humans were tested in navigation tasks in symmetrical environments, which allowed for the use of probe trials to assess navigation strategies (place or response) or impose them directly. The use of place strategies (on probe trials and place-training) was associated with increases in measures of VTE (reorientations and pausing) especially at high-cost decision points, similar to results from rodent studies. In contrast, response-strategies were associated with the development of efficient, stereotyped trajectories (consistent with model-free learning). These results support the identification of place- and response-strategies in human navigation with model-based and model-free learning, respectively, and demonstrate that VTE is specifically related to the use of place-strategies.

Keywords: vicarious trial-and-error, spatial memory, navigation strategy, virtual navigation, model-based behavior
\end{abstract}




\section{Vicarious trial-and-error is associated with the use of place-strategies in human virtual navigation}

It is well-known that effective navigation can be supported by separable strategies which depend on distinct neural systems. Place-strategies (allocentric, spatial, strategies which define goals by their location in the environment) depend on the hippocampus and dorsomedial striatum, while response-strategies (egocentric strategies which define goals by sequences of movements or stimulus-response relationships) depend on the dorsolateral striatum (Packard \& McGaugh, 1992; Packard \& McGaugh, 1996; Yin \& Knowlton, 2006). Rather than implementing a specific navigation strategy (allocentric versus egocentric), the involvement of these brain systems in navigation may be due to support for model-based behavior (where behavior is guided by internal, learned representations of the world) by the hippocampus and dorsomedial striatum, and model-free behavior (where behavior is produced in response to the immediate stimuli available) by the dorsolateral striatum (Khamassi \& Humphries, 2012; Redish, 2013; Vikbladh et al., 2019). Model-based behaviors are more flexible, but come with a computational cost as one searches through the available state space to find and evaluate potential actions. Model-free behaviors are less flexible, but more efficient, allowing one to take fast action based on the cached results of previous experience with actions taken in the current state. Identification of place- and response-strategies with model-based and model-free behavior, respectively, is also supported by work demonstrating that navigation based on distal cues (under conditions associated with hippocampal activity, Doeller, King, \& Burgess, 2008) and use of model-based behavior are correlated in normal individuals, and this relationship is disrupted after 
hippocampal lesions (Vikbladh et al., 2019). Also, in a spatial task requiring flexible planning hippocampal activity is related to model-based valuations (Simon \& Daw, 2011).

Consistent with the proposal that place-strategies typically represent model-based learning, vicarious trial-and-error (VTE) in rodents is enhanced under conditions which are hippocampally-dependent, or involve conflict between place- and response-strategies. During VTE, rats make a set of body movements between potential choices (Muenzinger, 1938; Tolman, 1948), and hippocampal ensembles represent potential future trajectories (Johnson \& Redish, 2007), and episodes of VTE may represent deliberation between options using a learned model of the world (reviewed by Redish, 2016). In dual-solution conditions (where place- or responsestrategies are not in conflict), rats show increased VTE early in training at high-cost decision points, and reduced VTE as behavior becomes automatized (Johnson \& Redish, 2007; van der Meer, M. A. \& Redish, 2009). When a place- or response-strategy is imposed (by using multiple starting locations, and defining the correct goal location according to the imposed strategy), rats show enhanced VTE during the use of place-strategies, when making errors, and in trials following errors (Schmidt, Papale, Redish, \& Markus, 2013). And, in hippocampal-dependent spatial memory tasks, hippocampal lesions do not abolish VTE, but disrupt the relationship between VTE and learning of goal locations (Bett et al., 2012).

In humans, there is less evidence that place-strategies are specifically associated with behaviors similar to rodent VTE or other behavioral correlates of deliberation. One recent study by Santos-Pata and Verschure (2018) trained humans in a multiple-T maze where placestrategies were rewarded. Measure of VTE (based on changes in the head orientation of the participants' avatar) paralleled findings in rodents: VTE measures decreased as participants 
learned to find each target successfully, and both higher dwell times and more variable head orientations were found at early, high-cost decision points.

Behavioral studies also support the proposal that response-strategies represent model-free learning. During dual-solution training on a multiple-T maze, both rats and humans show slow development of consistent, stereotyped trajectories, as would be expected for model-free learning (Schmitzer-Torbert, \& Redish, 2002; Schmitzer-Torbert, 2007). On the same task, humans who used response strategies on a probe trial also showed more consistent trajectories on trials before the probe (Schmitzer-Torbert, 2007). In rats, the slow development of consistent trajectories is paralleled by a gradual improvement of task representation by striatal neurons (van der Meer, M. A., Johnson, Schmitzer-Torbert, \& Redish, 2010).

To specifically test the relationship between human VTE and the use of place-strategies and between stable trajectories the use of response-strategies, I examined behavior under several conditions: 1) during probe trials conducted after an initial phase of dual-solution training, 2) during strategy training when place- or response-strategies were imposed, and 3) during transfer to a strategy (place or response) after an initial phase of dual-solution training. Measures of VTE used here have been characterized in an experiential foraging task (Huynh, Alstatt, Abram, \& Schmitzer-Torbert, 2020) which replicated several results from rodents in which deliberation was associated with increased VTE. Across the present studies, the expression of place-strategies during probe trials and strategy training was associated with VTE-like behaviors (reorientations) while response-strategies were associated with rapidly initiated, low-variability (stereotyped) behavior. 


\section{Results}

\section{Experiment 1}

Participants were tested in a symmetrical multiple-T maze (Figure 1A-B), using dualsolution training (where the starting location and target placements were fixed across trials). Results include data from several samples, in which participants learned to find one or two hidden targets, and who were tested in-person or who completed the task independently. All participants completed pretraining on a Barnes maze (Supplementary Figure 1A), and a subset (n =41) completed pre-training on the multiple-T maze. Probe trials were conducted twice (early and late in training) to assess navigation strategy by rotating the participants' starting position by $180^{\circ}$. I predicted that place-strategies would be associated increased VTE-like behavior, while response-strategies would be associated with stereotyped trajectories.

\section{Pre-training}

Results from pre-training demonstrated that the extramaze cues provided were sufficient to support place-learning (see example in Supplementary Figure 1B). By the end of Barnes maze training $64 \%$ of participants reached a criterion at least 2 correct choices in the final 3 trials. In separate ANOVAs, with block as a within-subjects factor and location of testing (in-person versus independent) as a between-subjects factor, there were significant main effects of block for both for both accuracy $\left(\mathrm{F}(5,335)=36, \mathrm{p}<0.001, \eta_{p}^{2}=0.35\right)$ and the size of the initial choice error $\left(\mathrm{F}(5,335)=22, \mathrm{p}<0.001, \eta_{p}^{2}=0.25\right.$, see Supplementary Figure 1C-D). There were no significant main effects or interactions with testing location ( $\mathrm{ps}>0.06, \eta_{p}^{2} \mathrm{~s}<0.052$ ). 


\section{Figure 1}

Experiment 1 environment and behavior
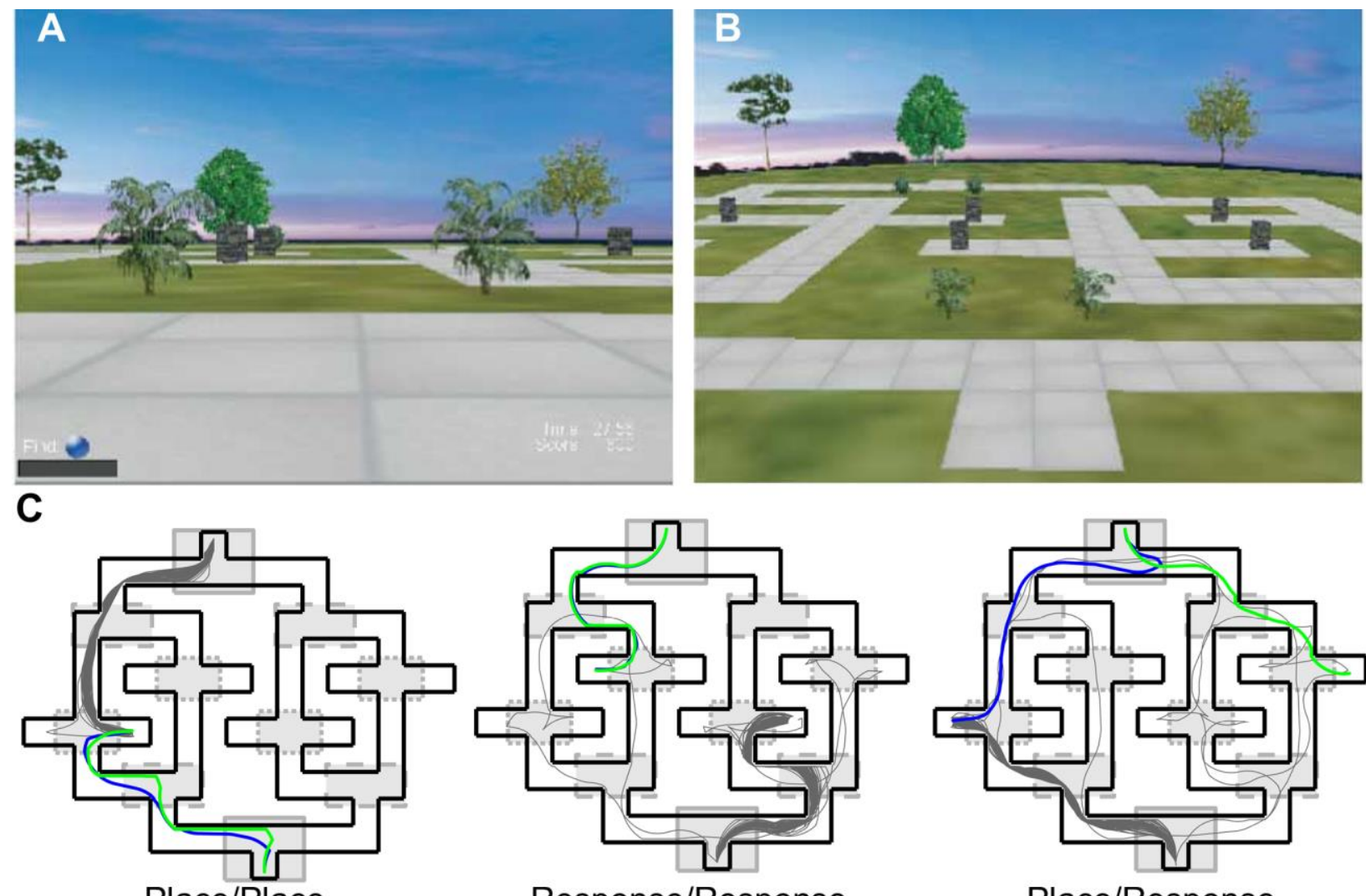

Response/Response

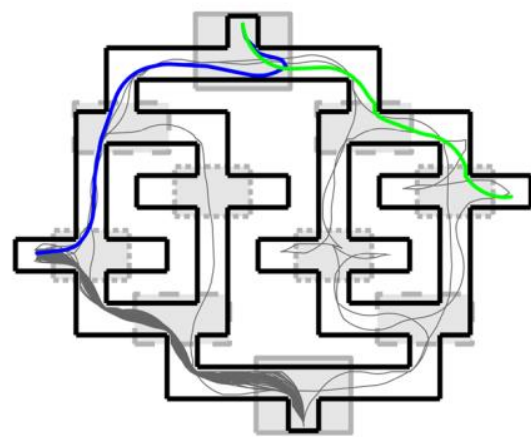

Place/Response

D

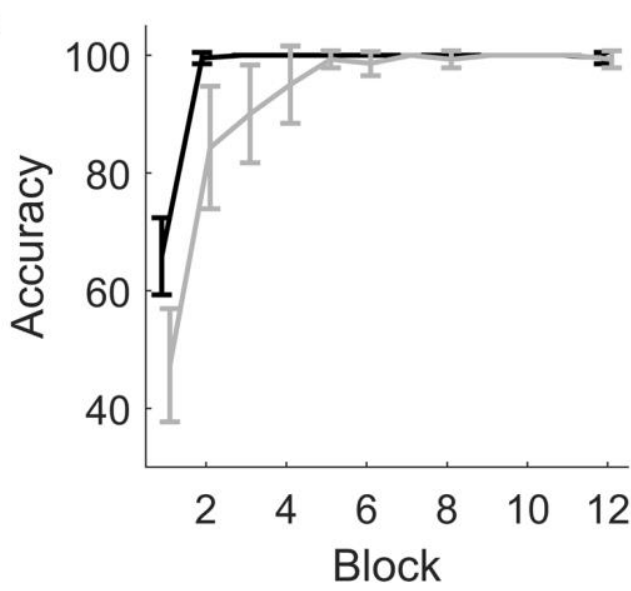

E

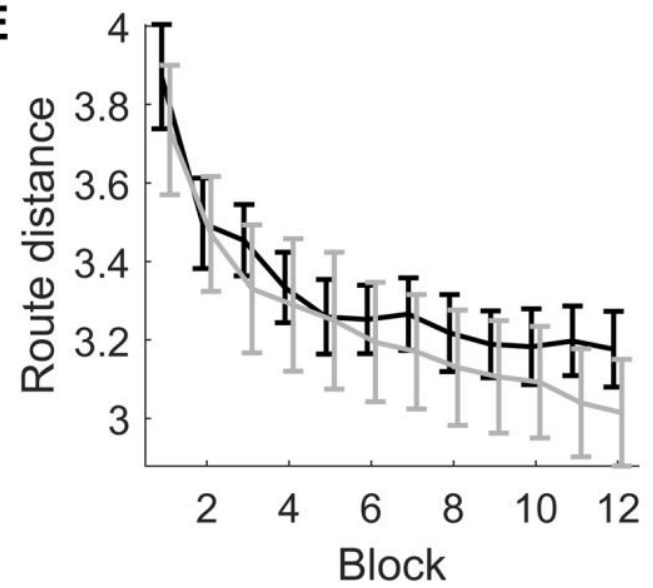

Note. A-B: First-person (A) and overhead (B) view of the multiple-T maze. C: Examples of participants who used a place strategy on both the early (blue) and late (green) probes (Place/Place, left, from session 10-26-2006-1264843), a response strategy on both probe trials (Response/Response, middle, from session 
10-09-2006-97359), and who switched from a place strategy on the early probe to a response strategy on the late probe trial (Place/Response, right, from session 10-09-2006-90265). Position data from normal training trials is shown in gray. Shaded areas indicate the choice point regions defined as the start arm (solid border), intermediate choice (dashed border), and final choice (dotted border). D-E: Multiple-T acquisition for the primary target in Experiment 1. A: Accuracy for first location chosen improved across the first several blocks of training, and slower when participants learned the location of two separate targets. B: Averaged route distance improved more slowly across the session. Data averaged for each block of five trials. Solid black $=$ training with one target, solid gray line $=$ training with two targets.

Multiple-T maze pre-training. Similarly, participants who completed pre-training on the multiple-T maze $(n=41)$ also improved significantly across training (Supplementary Figure 1E$\mathrm{F})$, where there was a significant main effect of block $\left(\mathrm{F}(5,195)=54, \mathrm{p}<0.001, \eta_{p}^{2}=0.58\right)$. Neither the main effect of testing location nor the block $\times$ testing location interaction $(\mathrm{ps}>0.08$, $\left.\eta_{p}^{2} \mathrm{~s}<0.075\right)$ were significant. By the final block of training, all participants had reached a criterion of at least 3 correct choices in the last 4 trials.

\section{Multiple-T acquisition}

During training on the multiple-T, participants quickly identified the location of the correct goal locations (Figure 1D), and more slowly adopted a stable route to their choice (Figure 1E). In separate ANOVAs with block as a within-subjects factor and number of targets (1 or 2) as a between-subjects factor, there were significant main effects of block for both measures, and a significant main effect of number of targets and the block $\times$ number of targets interaction for accuracy (ps $\left.<0.001, \eta_{p}^{2} \mathrm{~s}>0.11\right)$. Other effects were not significant ( $\left.\mathrm{ps}>0.1, \eta_{p}^{2} \mathrm{~s}<0.045\right)$. Post-hoc tests indicated that accuracy improved faster when participants were only learning to 
find one target, and both groups reached ceiling accuracy by the fourth block of training. Route distances continued to improve until the $8^{\text {th }}$ block of training, and did not depend on the number of targets.

\section{Path efficiency}

The slow development of stable routes is consistent with previous reports (SchmitzerTorbert, \& Redish, 2002; Schmitzer-Torbert, \& Redish, 2004; Schmitzer-Torbert, 2007), and may reflect optimization of the trajectory taken through the environment, or idiosyncratic solutions unique to individual. To determine if route stability was related to optimization, latency and distance overshoot scores were calculated for each trial as a percentage of excess time or distance, relative to the minimum achieved across the sample (to estimate the optimum for the multiple-T maze). Both measures improved across training (Supplementary Figure 2A-B) and in separate ANOVAs, there were main effects of block for both measures ( $\mathrm{ps}<0.001, \eta_{p}^{2} \mathrm{~s}>0.19$ ), and there were no main effects or interactions with the number of targets ( $\mathrm{ps}>0.18, \eta_{p}^{2} \mathrm{~s}<0.03$ ). Compared to the final block of training, post-hocs indicated that latency overshoot was elevated in the first two blocks and distance overshoot was elevated in the first block of training.

While latency and distance overshoot improved more quickly than route distance, participants who adopted more stable routes did have more efficient navigation. Within each block of training, participants with more similar routes (low route distances) achieved more optimal distances (in the third block, $\mathrm{r}(67)=0.27, \mathrm{p}=0.027$, across the other eleven blocks, $\mathrm{rs}>$ 0.35 , ps < 0.003) and more optimal latencies (across all blocks: rs $>0.60$, ps $<0.001$ ).

Participants who adopted more stable routes not only had more efficient navigation, but tended to converge to the same trajectories through the multiple-T maze. The last ten trials for 
the primary target were selected for each participant from trials in which the participant made their most common (egocentric) target choice. Using these trials, the average route distance was calculated within-participant and between-participants for every pair (to assess the overlap of the routes) who made the same egocentric target choice. For each pair, the higher of the two withinparticipant route distances (i.e. the worse of the two participants) is plotted against the betweenparticipant route distance in Supplementary Figure 2C. For pairs in which both participants adopted stable routes (pairs where the maximum within-participant route distance was below the sample median), the between-participant overlap did not differ from the within-participant

overlap, indicating participants had adopted similar trajectories (linear regression, $\beta$ slope $=1.1$, $95 \% \mathrm{CI}=[0.921 .19], \beta$ intercept $=-0.14,[-0.540 .26])$. In contrast, for pairs of participants where at least one pair member did not adopt a consistent route (pairs where the maximum within-participant route distance was above the median), the between-participant overlap tended to be higher than the within-participant overlap, indicating participants adopted distinct trajectories $(\beta$ slope $=1.30,[1.141 .46], \beta$ intercept $=-0.92,[-1.5-0.37])$. These results indicate that participants who adopted stable routes tended to converge on similar, optimal trajectories through the environment.

\section{Probe trials}

On probe trials, where participants' starting locations were rotated $180^{\circ}$, both place- and response-strategies were observed (see examples in Figure 1C). Participants who did not receive familiarization with maze (multiple-T maze pre-training) did not show any place strategies. 
Figure 2

Probe trial performance in Experiment 1
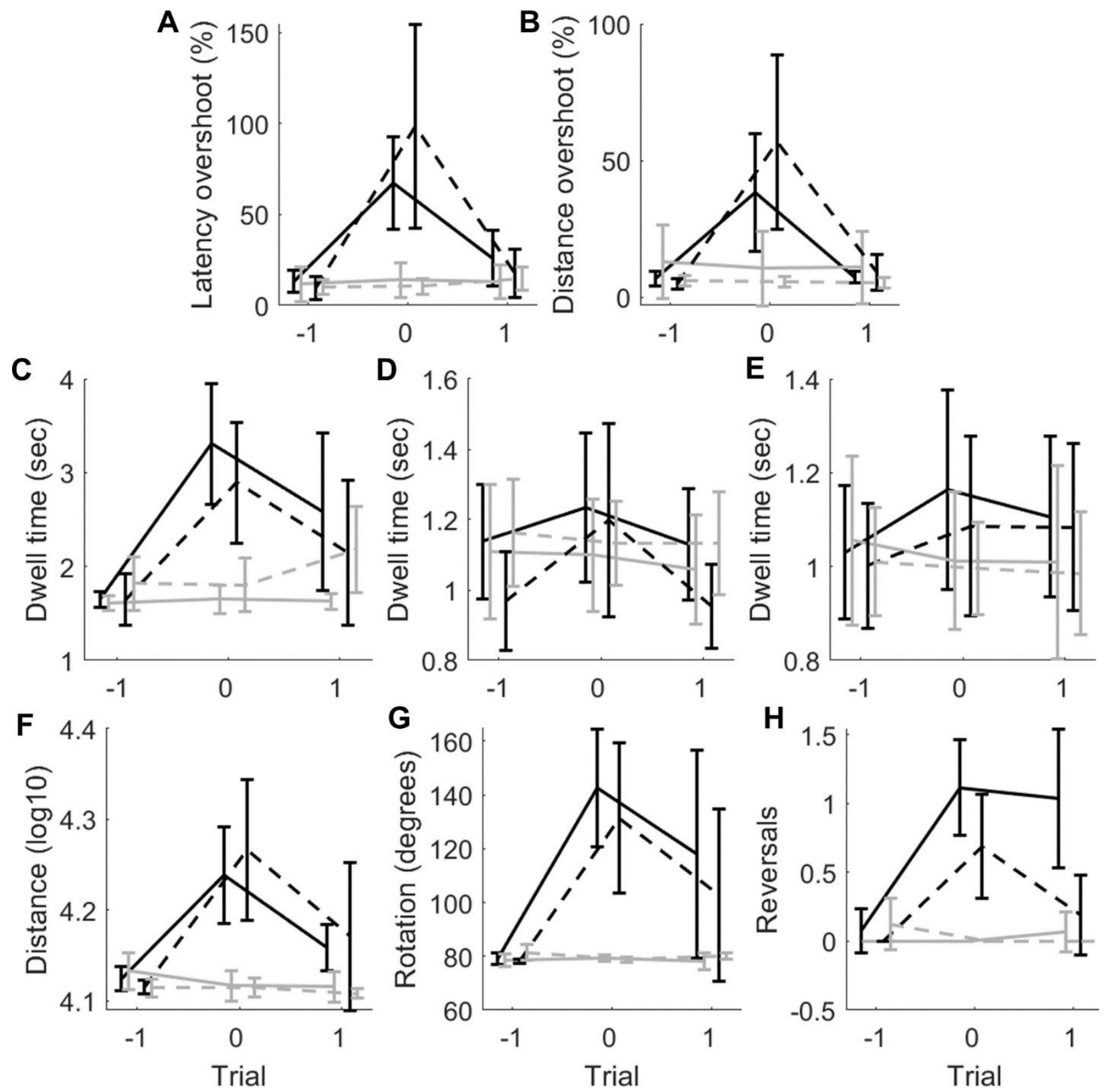

Note. On probe trials, place-strategies were associated with longer latencies and distance travelled $(A-B)$, longer dwell times in the start arm $(C)$, compared to the intermediate $(D)$ and final choice $(E)$. In the start arm, place-strategies were associated with longer distanced travelled $(F)$, greater rotation $(G)$, and more reversals of rotation direction $(H)$ on probe trials and in some cases on the subsequent dual-solution trial. Performance plotted for the probe trial (0) and the preceding (-1) and following (+1) trials for participants 
who made place-choices (black lines) or response-choices (gray lines) on early (solid) and late (dashed) probe trials.

While the Barnes maze results indicated that extramaze cues were sufficient for place-learning, it appears that participants disregarded such cues by default after transfer to the multiple-T maze. Analyses of probe trials were thus limited to samples using multiple-T maze pre-training $(\mathrm{n}=$ 41). Performance on probe trials for each sample is given in Table 1.

\section{Table 1}

Choices made on early and late probe trials for each sample in Experiment 1.

\begin{tabular}{|c|c|c|c|c|c|}
\hline \multirow[b]{2}{*}{ Tested } & \multirow{2}{*}{$\begin{array}{l}\text { multiple-T } \\
\text { pre-training }\end{array}$} & \multirow[b]{2}{*}{ Early probe } & \multicolumn{3}{|l|}{ Late probe } \\
\hline & & & Place & Response & Other \\
\hline \multirow[t]{3}{*}{ Independent } & No & Place & 0 & 0 & 0 \\
\hline & & Response & 0 & $23(88 \%)$ & 0 \\
\hline & & Other & 0 & $3(12 \%)$ & 0 \\
\hline \multirow[t]{3}{*}{ Independent } & Yes & Place & $5(24 \%)$ & $6(29 \%)$ & 0 \\
\hline & & Response & $1(5 \%)$ & $9(43 \%)$ & 0 \\
\hline & & Other & 0 & 0 & 0 \\
\hline \multirow[t]{3}{*}{ In-person } & Yes & Place & $10(50 \%)$ & $4(20 \%)$ & $1(5 \%)$ \\
\hline & & Response & 0 & $5(25 \%)$ & 0 \\
\hline & & Other & 0 & 0 & 0 \\
\hline
\end{tabular}

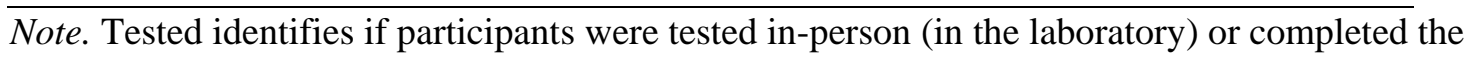
experiment independently (personal computer or public computer lab). Multiple-T pretraining was conducted after Barnes maze training for two samples. 
A total of 15 (37\%) participants used a place-strategy on both probe trials, while 14 $(34 \%)$ used a response-strategy on both probe trials. Eleven participants switched between placeand response-strategies, with the majority $10(91 \%)$ switching from a place-strategy early to a response-strategy late in training, a difference which was significant more common than switches from response- to place-strategies $\left(\chi^{2}(1, N=11)=7.4, p=0.007\right)$, and consistent with previous research (Schmitzer-Torbert, 2007).

While the shift from place- to response-strategies with extended training paralleled the slow development of a stable route, there was no such relationship between probe-trial strategy and dual-solution performance, contrary to my previous report (Schmitzer-Torbert, 2007). In the block before the probe, accuracy did not differ by strategy for the early (place: $100.0 \%, \mathrm{SD}=$ 0.0 , response: $98.7 \%, \mathrm{SD}=5.2, \mathrm{t}(39)=1.33, \mathrm{p}=0.19,95 \% \mathrm{CI}=[-0.70,3.4])$, or the late probe trial (place: $100.0 \%, \mathrm{SD}=0.0$, response: $99.2 \%, \mathrm{SD}=4.1, \mathrm{t}(38)=-0.8, \mathrm{p}=0.42,95 \% \mathrm{CI}=[-$ 1.24, 2.91]). Similarly, there were no significant differences in the route distance in the block preceding the early (place: $\mathrm{M}=3.50(\mathrm{SD}=0.36)$, response: $\mathrm{M}=3.49, \mathrm{SD}=0.39, \mathrm{t}(39)=0.123$, $\mathrm{p}=0.903,95 \% \mathrm{CI}=[-0.23,0.26])$ or the late probe trial (place: $\mathrm{M}=3.36, \mathrm{SD}=0.32$, response: $\mathrm{M}=3.58, \mathrm{SD}=0.38, \mathrm{t}(38)=-1.935,, \mathrm{p}=0.060,95 \% \mathrm{CI}=[-0.45,0.01])$, where participants who made response-choices tended to have more variable routes. Similar results were obtained using ANOVAs for each measure, with block as a within-subjects factor and probe-choice group (Place/Place, Response/Response, or Place/Response) as a between-subjects factor, which found no main effects or interactions of probe-choice group ( $\mathrm{ps}>0.19, \eta_{p}^{2} \mathrm{~s}<0.08$ ). 


\section{Behavior during probe trials}

While dual-solution behavior did not predict probe-trial choices, behavior on probe trials did strongly discriminate between the strategies. Relative to the trials preceding and following each probe, participants who made place-choices had higher latency overshoot (Figure 2A), and distance overshoot scores (Figure 2B), while participants who made response choices did not show a change in their distance or latency overshoot scores (route distance scores were not examined, as participants who made place-choices often made egocentric choices which were not repeated). Separate ANOVAs for each dependent measure and probe trial (early and late) were conducted with trial (relative to probe: $-1,0,+1$ ) as a within-subjects factor and probe choice (place or response) as a between-subjects factor. For both probe trials, there were significant trial $\times$ probe choice interactions for both latency and distance overshoot $(\mathrm{ps}<0.006$, $\left.\eta_{p}^{2} \mathrm{~s}>0.12\right)$. Post-hocs indicated participants who made place-choices had significantly higher latency and distance overshoots relative to the preceding and following trial and to participants who made response-choices.

Place-choices were associated with less efficient navigation, and this result was consistent with the expectation that place-strategies would involve increased deliberation. Examining dwell times in each of the three decision points (shaded areas in Figure 1C, showing the initial start arm, intermediate choice, and final choice zone), place-choices were associated with longer dwell times selectively in the initial start arm (Figure 2C-E). Using separate ANOVAs by decision point and probe trial, there were significant trial $\times$ probe choice interactions for the initial start arm on both probe trials, as well as a main effect of trial for both probes and of probe choice for the early probe (ps $<0.006, \eta_{p}^{2} \mathrm{~s}>0.13$ ), while other main effects and interactions were not significant $\left(\mathrm{ps}>0.06, \eta_{p}^{2} \mathrm{~s}>0.08\right)$. Post-hocs indicated that dwell times 
in the start arm were significantly higher for participants who made place-choices, compared to the preceding trial (-1), and to participants who made response-choices. Following the probe trial, dwell times in the start arm were intermediate between the earlier trials $(-1,0)$ for participants who made place-choices. These results indicate that the use of place-strategies was selectively associated with longer dwell times at a high-cost decision point.

\section{VTE-like behavior in the start arm}

Place-choices were also associated with VTE-like behaviors, where participants made one or more reorientations before exiting the start arm (Figure 1C, left and right place-choice examples). VTE-like behaviors (distance travelled, rotation, and pausing) were characterized using a set of measures developed for a translational foraging task (Huynh et al., 2020) applied to behavior in the start arm (up to the first exit on each trial). In separate ANOVAs for each dependent measure and probe, there were significant trial (relative to probe, $-1,0,1) \times$ probe choice (place, response) interactions for the total distance travelled, total angular rotation, and number of reversals of rotation direction while in the start arm for both probe trials (Figure $2 \mathrm{~F}-\mathrm{H}$, ps $\left.<0.04, \eta_{p}^{2} \mathrm{~s}>0.079\right)$, but not for pausing before exiting the start arm (ps $\left.>0.21, \eta_{p}^{2} \mathrm{~s}<0.041\right)$. Post hocs indicated that place-choices were associated significantly longer distances travelled, more total angular rotation, and more rotation reversals compared to the previous trial and to participants who made response-choices. On the trial following the probe $(+1)$ these measures tended to return to levels observed before the probe $(-1)$, but total angular rotation remained elevated on both probe trials, and rotation reversals they remained significantly after the first probe and were intermediate for the late probe. These results demonstrate that during probe 
trials, participants who express a place-strategy show several measures consistent with VTE on the probe trial, and some of these measures persist to the subsequent dual-solution trial.

\section{Experiment 2}

The results of Experiment 1 demonstrated that VTE-like behaviors in human virtual navigation were specifically associated with the use of place-strategies on probe trials, and were selectively enhanced at a high-cost decision point, consistent with results in rats and humans (Johnson \& Redish, 2007; Santos-Pata \& Verschure, 2018; van der Meer, M. A. \& Redish, 2009). Participants also slowly optimized their routes, adopting stereotyped trajectories across dual-solution training. There was a parallel shift to an increased use of response-strategies late in training. These results are broadly consistent with the proposal that place-strategies represent model-based behavior (allowing for flexible, but computationally costly, behaviors) while response-strategies represent model-free behavior (allowing for rapid execution of behaviors based on cached experience). However, behavior during dual-solution training was not predictive of probe-trial behavior (except that some VTE measures remained elevated following the probe), and so it is inconclusive from these data if VTE and route stability are related to the execution of place- and response-strategies, respectively.

To determine if VTE and the development of a stable route are specifically associated with the use of place- and response-learning, respectively, participants were tested in a new environment while place- or response-training was imposed. Training was conducted on a virtual island where paths to goal locations were defined by obstacles (rocks/boulders) placed apparently at random, but which were symmetrical around each $90^{\circ}$ rotation (Figure $3 \mathrm{~A}-\mathrm{C}$ ). Strategy training was imposed by varying the starting location across trials, and placing targets 
according to one strategy (place or response). Participants learned to find one hidden target object using a place-strategy and to find a separate object using a response-strategy, trained in successive phases, with the order of training randomized. I predicted that success during placetraining would be associated with faster learning overall, and higher levels of VTE-like behaviors, while response-training would be associated with the development of stable, efficient routes.

\section{Acquisition}

Across strategy training, participants achieved similar accuracies for both place- and response-training (Figure 4A). In an ANOVA analyzing accuracy with gender and order of training (place $\rightarrow$ response, response $\rightarrow$ place) as between-subjects factors, and strategy (place, response) and block (1-4) as within-subjects factors, there was a significant main effect of block and strategy $\times$ block interaction $\left(\mathrm{ps}<0.028, \eta_{p}^{2} \mathrm{~s}>0.30\right)$. No other interaction or main effect was significant (all ps $>0.1, \eta_{p}^{2}<0.027$ ). Post-hocs revealed that accuracy improved significantly from block 1 to 2 for place-training, and from block 3 to 4 for response-training, indicating that place training was associated with somewhat more rapid improvements in accuracy.

While there was a significant improvement in accuracy for both strategies, many participants did not fully acquire the task by the final block of training, suggesting that this task was more difficult than Experiment 1. In the final block of training, 60/102 participants (59\%) reached criterion performance of at least 3 correct choices for the last 5 trials in place-training, compared to $64 / 102(63 \%)$ in response-training. Across the sample, 14 participants failed to acquire either strategy, 28 acquired only the place-strategy, 24 acquired only the response- 


\section{Figure 3}

Environment used in Experiments 2 and 3 (pilot) and examples of behavior from Experiment 2
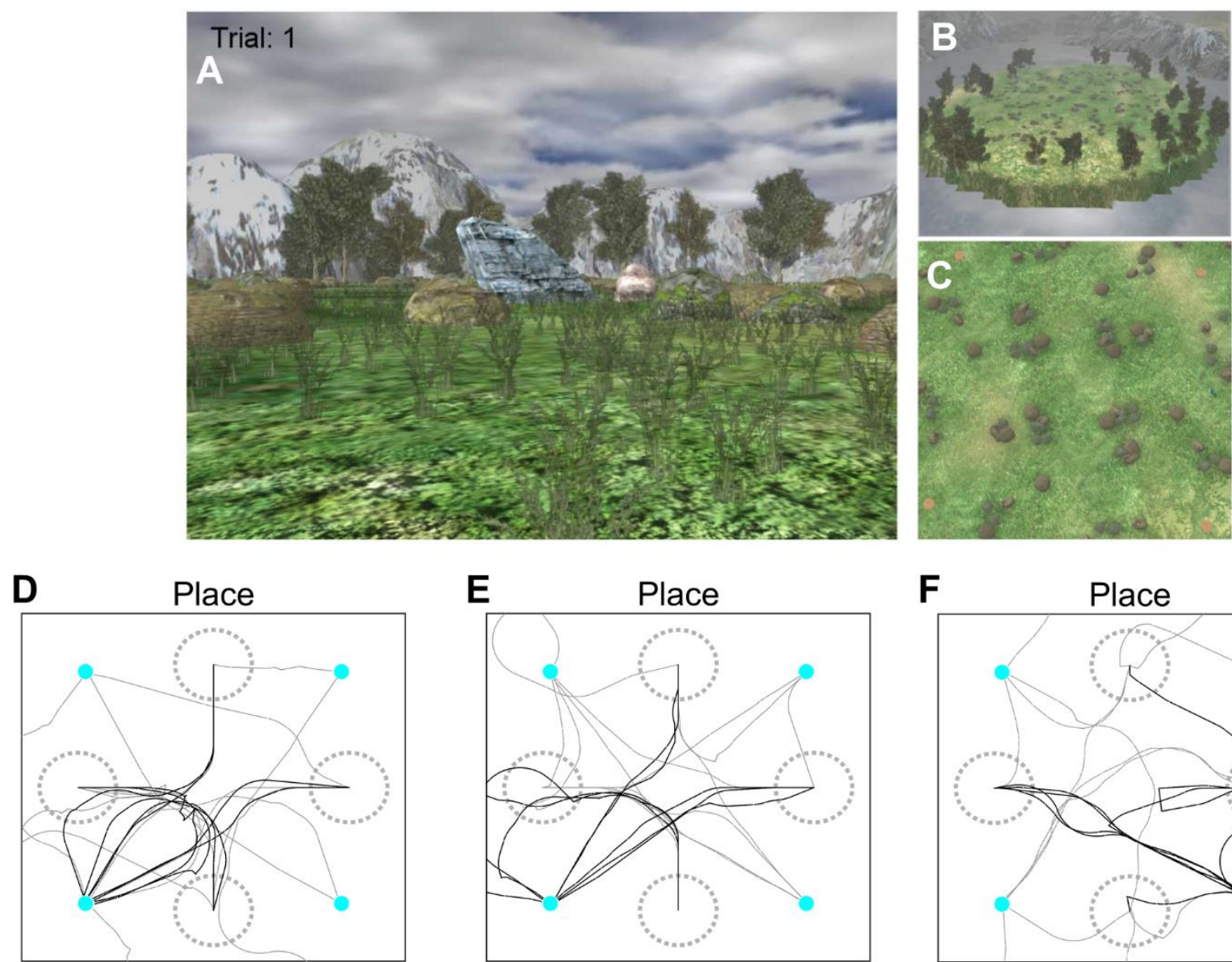

$\mathbf{F}$
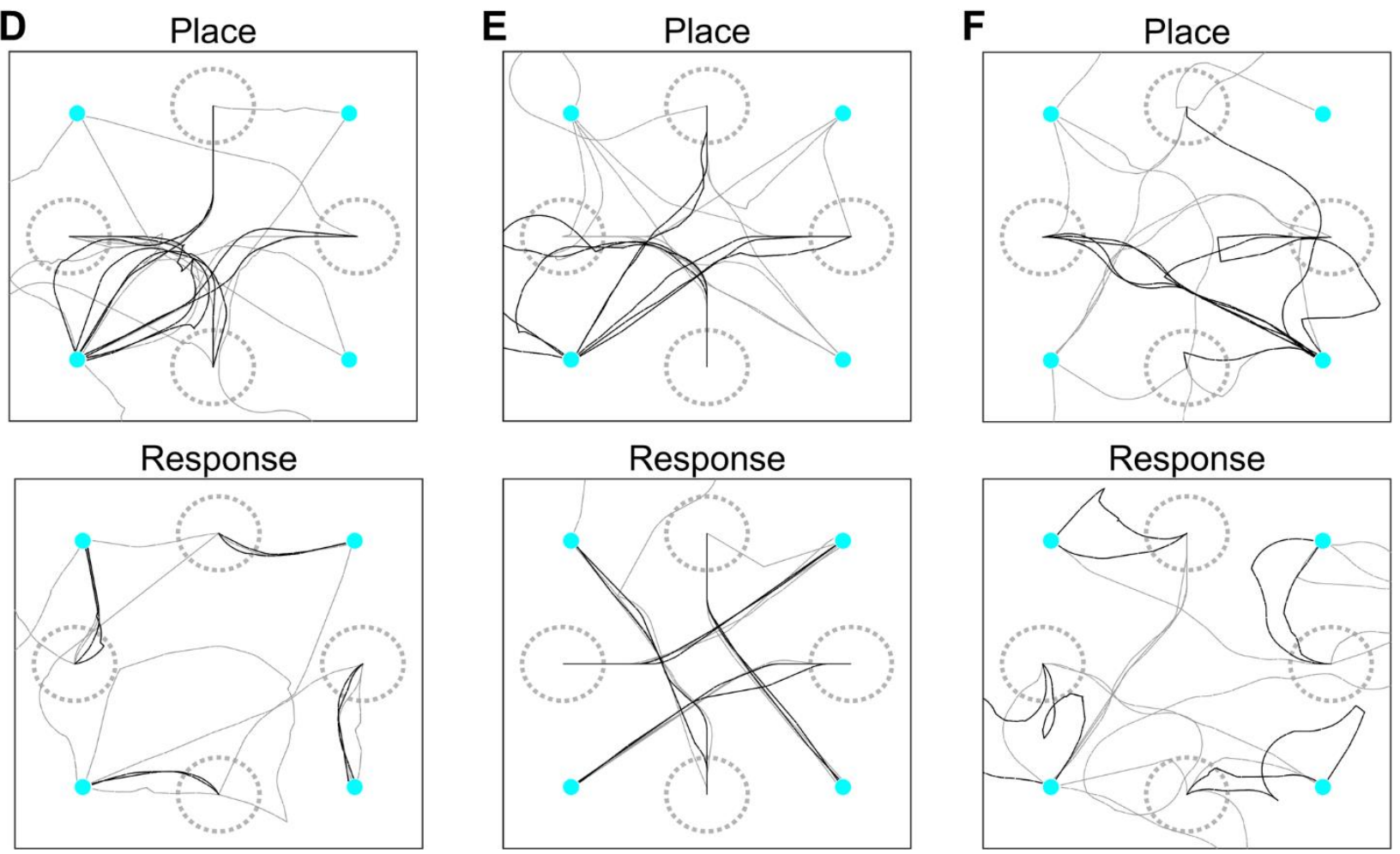

Note. A: First-person perspective view. B: Overhead view of the island. $C$ : View of the goal locations (beige circles) and obstacles (rocks). The rock and cylinder pattern was symmetrical around each $90^{\circ}$ rotation. D-E: Examples of navigation behavior from three participants who successfully acquired both navigation strategies. In each plot, place training is shown in the top panel, and response-training in the 
bottom panel. Grey points $=$ early training. Black points $=$ final eight accurate choices. Cyan circles indicate the location of the four targets, dashed gray lines indicate the limits of the starting zone for each starting location. Data from: (left) 2009-2-25-08-25-10-2777, (middle) 2009_1Maze_21-2009-3-13-1434-59-1484, and (right) 2009_1Maze_02-2009-3-13-10-15-29-5162. 


\section{Figure 4}

Task acquisition in Experiment 2
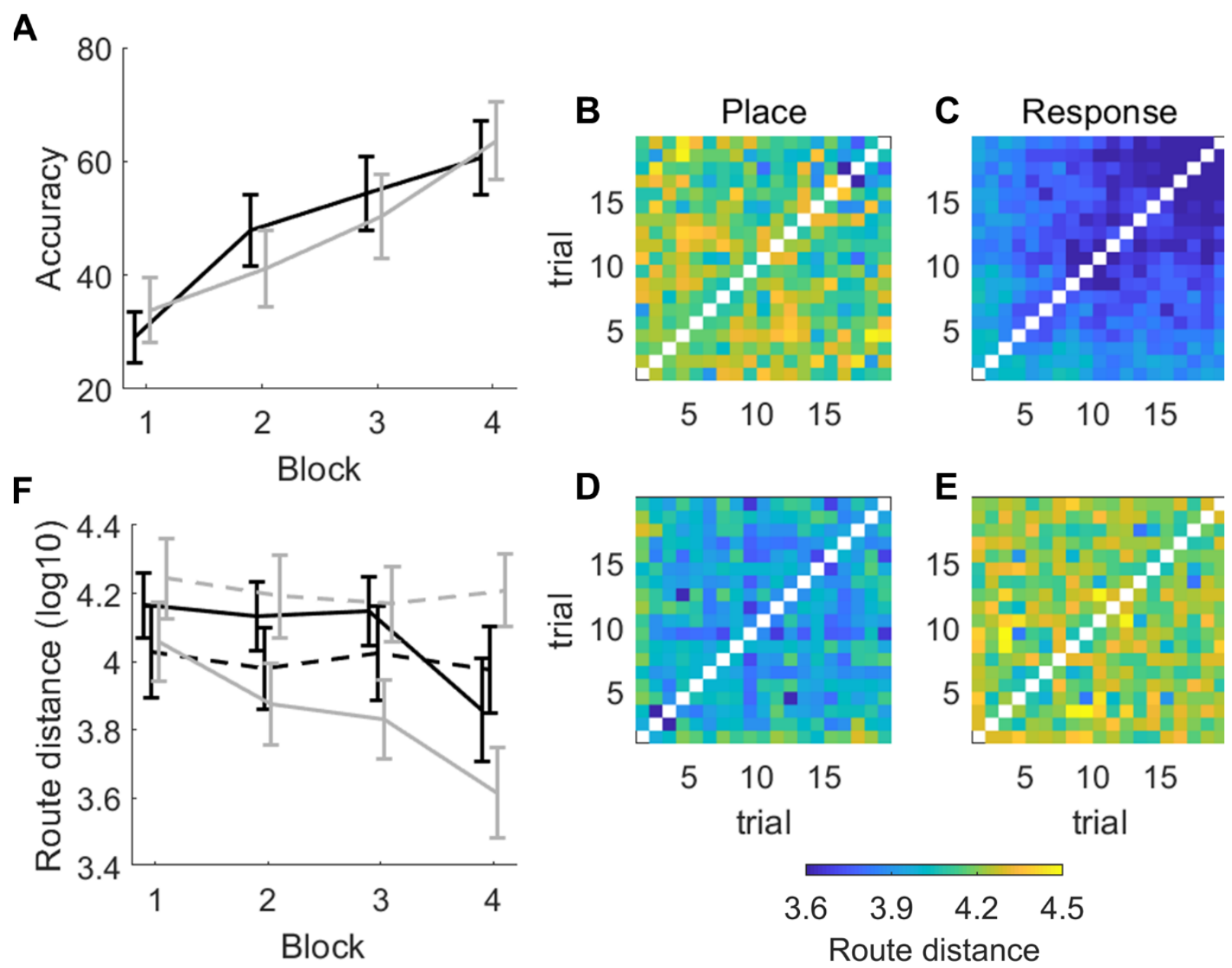

Note. A: Accuracy improved at similar rates across training, though somewhat faster for place-training (black line) compared to response-training (grey line). B-E: Average route distance matrices. Color indicates the average route distance between every pair of trials. B-C: Participants who successfully acquired the strategy. D-E: Participants who failed to acquire the strategy. Place-training is shown in B and D, Response-training in C and E. Participants who acquired the imposed strategy (solid lines) showed significant improvement across the session, compared to those who did not acquire the strategy (dashed lines), participants who acquired the response-strategy (grey) successfully showing more similar routes than participants who acquired the place-strategy (black). Lines indicate means, and errorbars indicate $95 \%$ confidence intervals. 
strategy, and 36 acquired both strategies, indicating that success on one strategy was not predictive of success on the alternate strategy $\left(\chi^{2}(1, \mathrm{~N}=102)=0.47, \mathrm{p}=0.49\right)$.

\section{Relationship of behavioral measures to place- and response-training}

While success during place- and response-training was similar across the sample, behaviors during navigation strongly discriminated between the imposed strategies. Participants who successfully acquired the response-strategy showed a strong decrease in their route distance scores (adopting stable routes, see examples in Figure 3D-E), compared to those who failed to acquire the response-strategy (Figure 3F), and to those who succeeded during place-training (Figure 4B-F), and a similar pattern was obtained for reaction times (Supplementary Figure 3D). Success during response-training was also associated with more efficient navigation (taking more direct routes from the starting location to the goal, with less excess rotation, see Supplementary Figure 3A-B), while successful place-training was associated with less efficient navigation (less direct paths from the starting location to the goal, see examples in Figure 3D-F), higher excess rotation, and more reversals of rotation direction (Supplementary Figure 3C).

Separate ANOVAs were used to analyze each dependent measure and strategy, with block (1-4) as a within-subjects factor, and with outcome (successful/unsuccessful acquisition of the imposed strategy), gender, and order of training as between-subjects factors. Several main effects or interactions with order of training were obtained for both place- and response-training. The general pattern of these results were that for each measure, scores were lower when the imposed strategy was the second one learned, and thus likely represent general improvements related to learning the task (movement controls and the general layout of the environment). Some gender differences were also obtained (e.g. males had faster reaction times than females, 
significant main effects of gender for both place- and response-training, ps $<0.001, \eta_{p}^{2}>0.12$ ), but no gender interactions with outcome were obtained.

During place-training, there were main effects of outcome for distance overshoot, excess rotation, and rotation reversals ( $\mathrm{ps}<0.049, \eta_{p}^{2} \mathrm{~s}>0.04$ ), main effects of block for route distance, reaction time, and pausing ( $\mathrm{ps}<0.043, \eta_{p}^{2} \mathrm{~s}>0.028$ ), and significant interactions of outcome $\times$ block for route distance and pausing $\left(\mathrm{p}<0.037, \eta_{p}^{2} \mathrm{~s}>0.029\right)$. For the interactions, post-hoc tests indicated that route distance scores decreased significantly in the fourth block those who succeeded in place-training, and did not change significantly for those who failed to acquire the place-strategy. Participants who failed to acquire the place strategy were also more likely to pause in the first block of place-training (Supplementary Figure 3E). These results indicated that participants who acquired the place-strategy had less efficient navigation across training, and were more likely to make corrections (rotation reversals), though their routes became somewhat more similar by the end of training.

For response-training, there were significant main effects of outcome for distance overshoot, excess rotation, and reaction time (ps $<0.026, \eta_{p}^{2} \mathrm{~s}>0.050$ ), main effects of block for route distance, distance overshoot, reaction time, and pausing ( $\mathrm{ps}<0.020, \eta_{p}^{2} \mathrm{~s}>0.034$ ), and significant interactions of outcome $\times$ block for route distance, excess rotation and reaction time $\left(\mathrm{p}<0.003, \eta_{p}^{2} \mathrm{~s}>0.049\right)$. Successfully acquiring the response-strategy was associated with faster reaction times, more similar routes, and more efficient navigation across all four blocks of training, and with a reduction in route distance scores, reaction time and excess rotation across response-training. 


\section{Experiment 3}

The results of Experiment 2 were broadly consistent with those of Experiment 1, demonstrating that successful place-training was associated with some VTE-like behaviors (reorientations) and with the use of more variable, less efficient navigation. Successful responsetraining was also associated with the development of efficient, stereotyped routes initiated at short latencies. Both patterns of results are consistent with expectations for model-based and model-free learning, respectively. However, while Experiment 1 found that VTE was enhanced during the use of place-strategies (on the probe and following trial), route stability and navigation efficiency were strongly related to the use of response-strategies on the probe trial. It could be the case, however, that such a relationship would have emerged if participants had been given the opportunity to express their navigation strategy on more than just the two probe trials (if, similar to Experiment 2, strategy training had been included).

Experiment 3 addressed this question by combining initial dual-solution training with subsequent strategy training to assess the relationship between the navigation strategy (assessed during the probe trial) and measures of VTE and route stability (on the probe trials and during strategy training). Training was conducted on a circular platform using a Barnes maze (Figure 5A-B), where participants learned to find one or two targets during an initial phase of dualsolution training. Probe trials were conducted on a randomly selected trial (to assess shifts from place- to response-strategies with extended training). After the probe trial, participants were moved to strategy training (either place or response, randomly selected). I predicted that the use of place-strategies (on the probe trial and place-training) would be associated with greater VTElike behavior, while the use of response-strategies would be associated with the development of stable, stereotyped trajectories. An initial pilot was conducted using the environment from 


\section{Figure 5}

Experiment 3 environment and examples of behavior

A

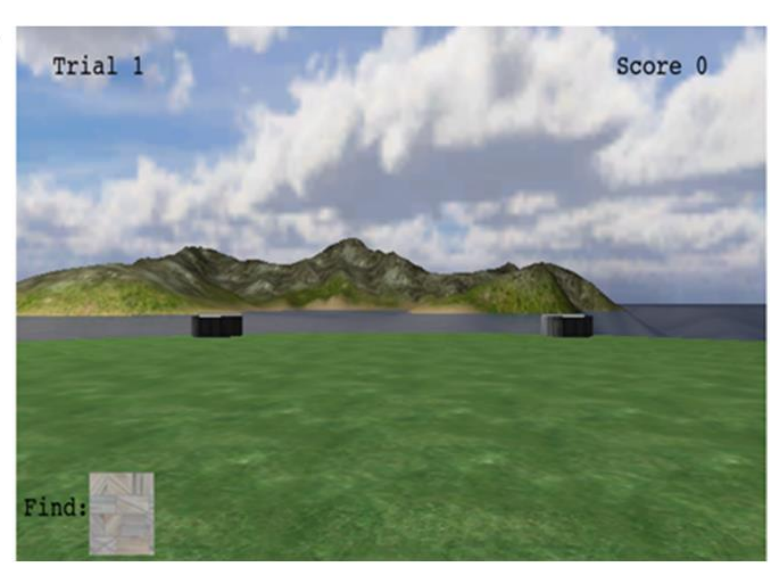

C

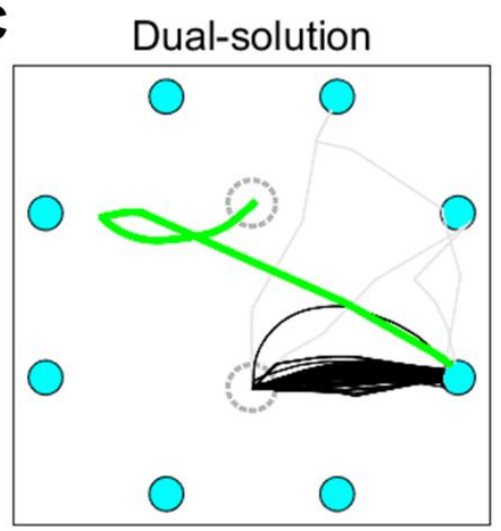

Place

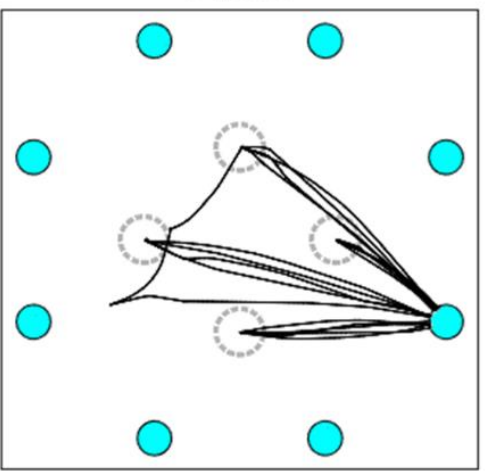

D

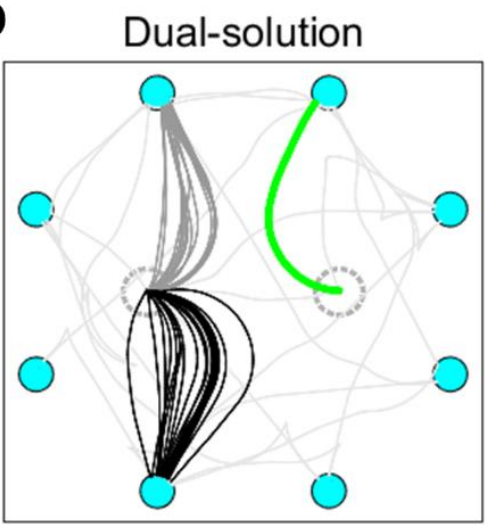

Response

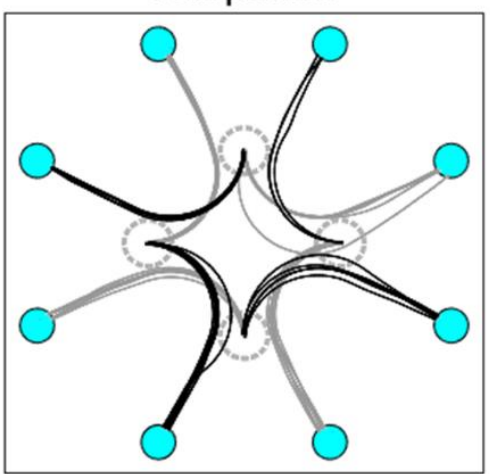

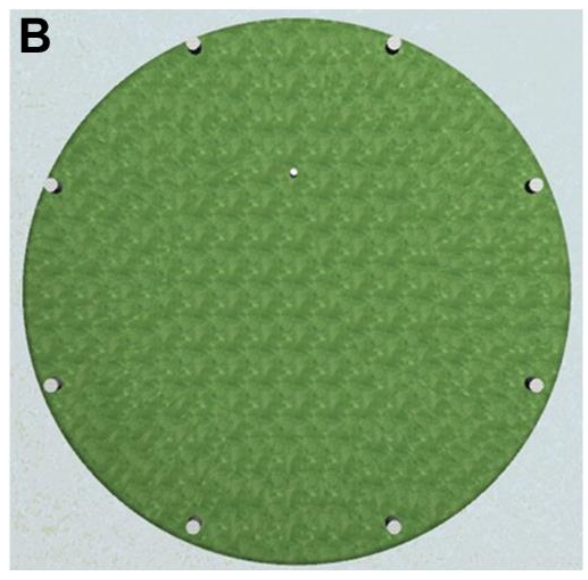

E

Eual-solution

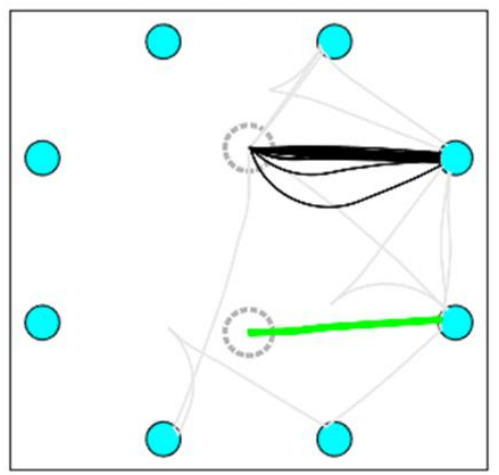

\section{Place}

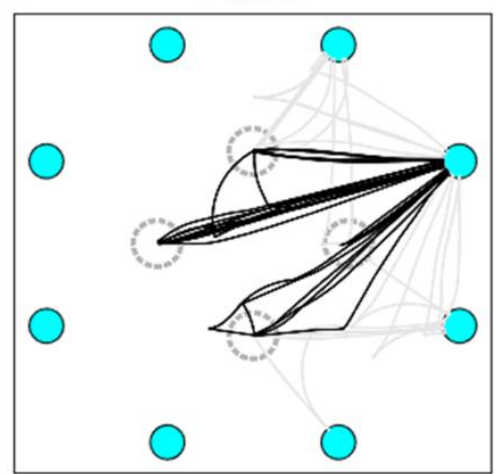

Note. A: First person view of the environment. B: Overhead view of the Barnes maze, indicating the location of each cylinder. Small white dot above the center of the task indicates one starting location. $C$ $D$ : Examples of participants in the Early-switch/Place-training groups. Top panel in each plot shows dualsolution training, and the bottom panel shows transfer to the imposed strategy. Black lines indicate paths 
up to the first location chosen on correct trials for the target used for the probe trial. Thick grey lines indicate paths up to the first location chosen for the second target (for participants who were trained to find two targets, $D$ ). Thin grey lines show incorrect trials. Cyan circles indicate the location of the eight target locations, dashed gray lines indicate the locations of the four starting locations. Green points indicate the probe-trial. (C: session 2010-09-20-18-37-1029013, D: session 2010-09-21-17-03-1013545, E: session 2010-09-21-16-46-1007950).

Experiment 2, the results of which were largely consistent with those reported here for Experiment 3 (see Supplementary Online Material).

\section{Dual-solution acquisition}

Across dual-solution training, accuracy increased across the first 10-20 trials (Figure 6A), more rapidly when participants were learning the location of only one target. Accuracies for participants whose probe trial occurred after the $30^{\text {th }}$ trial $(n=165,63$ females $)$ were analyzed using an ANOVA with block (1-6) as a within-subjects factor, and with gender and number of targets (1 or 2) as between-subjects factors. There were significant main effects of block, number

of targets, and the block $\times$ number of targets interaction (ps $\left.<0.021, \eta_{p}^{2} \mathrm{~s}>0.016\right)$. No other main effect or interactions were significant ( $\mathrm{ps}>0.38, \eta_{p}^{2} \mathrm{~s}<0.007$ ). By the sixth block of training, 81 of 88 (92\%, 32/34 females, 49/54 males) participants trained to find a single target had reached a criterion of $60 \%$ accuracy, compared to 64 of 89 (72\%, 24/33 females, 40/56 males) participants trained to find two targets. 


\section{Probe trials}

The majority of participants who reached criterion performance by the probe trial (accuracy $\geq 60 \%$ in the preceding five trials) made place- $(60 / 208,29 \%$, see example in Figure 5C) or response-choices (76/208, 37\%, see example in Figure 5D), which was significantly higher than would be expected by chance $\left(12.5 \%\right.$ expected for each, $\chi^{2}(2, N=208)=186, p<$ 0.001). A sizable proportion of the other probe trial choices likely represented attempts to use a place-strategy: $76.4 \%$ of these choices were adjacent to the place-choice location (error scores relative to the place-defined location $= \pm 1$, or $\mid$ error $_{\text {place }} \mid=1$, see example in Figure 5E), compared to $16.7 \%$ of choices which were adjacent to the response-choice location (|error response| $=1)$. Across the sample, $114 / 208(55 \%)$ of participants made a place-choice or adjacent location on the probe trial, compared to $89 / 208$ (43\%) of participants who made a response-choice or adjacent location, demonstrating a significant bias towards the strategy-defined locations on the probe trial $\left(37.5 \%\right.$ expected for each, and $25 \%$ for the "other" locations, $\chi^{2}(2, N=208)=61, p<$ $0.001)$.

Consistent with Experiment 1, participants who made place-choices on the probe trial had more variable trajectories, used inefficient routes, were likely to make reorientations, and to pause (Figure 6B, Supplementary Figure 6D-G). Using separate ANOVAs with probe choice (place \pm 1 or response \pm 1 ) and gender as between-subjects factors, and trial (relative to the probe: -1 or 0 ) as a within-subjects factor, there were significant probe choice $\times$ trial interactions for distance scores, excess rotation, number of rotation reversals and probability of pausing (ps < $0.034, \eta_{p}^{2} \mathrm{~s}>0.028$ ), while there were no main effects or interactions with gender ( $\mathrm{ps}>0.078$, $\left.\eta_{p}^{2} \mathrm{~s}<0.019\right)$. 


\section{Figure 6}

Experiment 3 behavioral measures
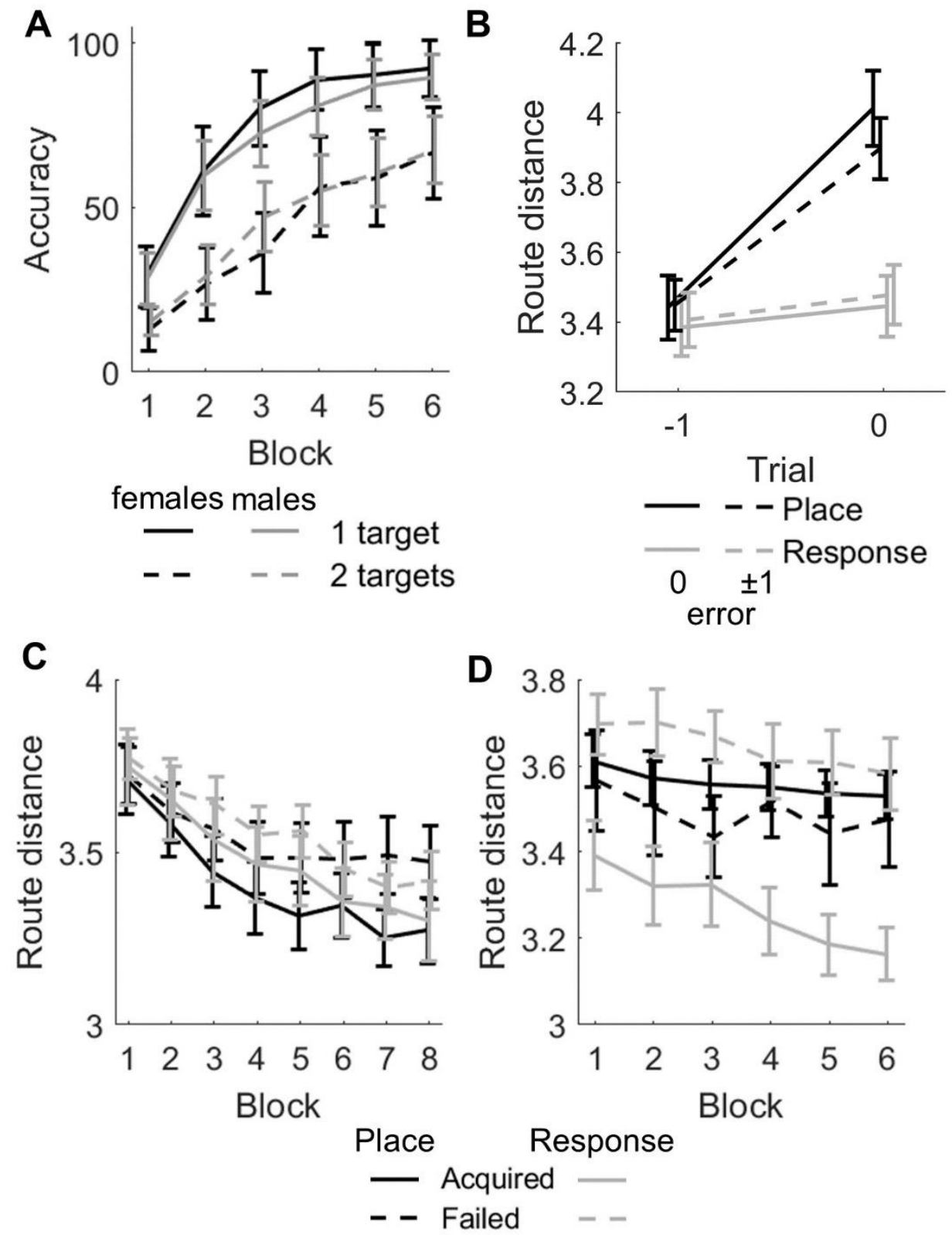

Note. A: Accuracy during dual-solution training improved across the first five blocks of training, at similar rates for females and males, and more slowly when participants were trained to find two separate targets, compared to one. Black lines $=$ females, gray lines $=$ males, solid lines $=$ trained to find one target, dashed lines $=$ two targets. B: On probe trials, participants who made place-choices $\left(\right.$ error $\left._{\text {place }}=0\right)$ 
and/or chose the adjacent locations (|error place $\leq 1)$ used more variable routes on the probe trial compared to those who made response-choices $\left(\right.$ error $_{\text {response }}=0$ or $\mid$ error $\left._{\text {response }} \mid \leq 1\right)$. Black lines $=$ place, gray lines $=$ response, solid lines $=$ error $_{\text {strategy }}=0$, dashed lines $=\mid$ error $_{\text {strategy }} \mid \leq 1$. C: During dual-solution training, participants who would succeed during strategy training (place or response) tended to have more similar routes, before transfer. D: After transferring to strategy training, participants who acquired the responsestrategy had more similar trajectories, while participants who acquired the place-strategy had higher more variable routes scores, compared to participants who failed to acquire the imposed strategy. Black lines $=$ transferred to place-training, gray lines $=$ transferred to response-training, solid $=$ acquired the imposed strategy, dashed = failed to acquire the imposed strategy. Lines indicate means and errorbars indicate $95 \%$ confidence intervals.

Across training, there was no evidence for a shift in the likelihood of using a place- or response-strategy (Supplementary Figure 6A). When probe trials (falling between trial 11 and 91) were grouped into four equal sized bins, there was no significant difference in the proportion of place-strategies observed across the four bins $\left(\chi^{2}(3, \mathrm{~N}=203)=3.0, \mathrm{p}=0.39\right)$. However, the proportion of participants who reached criterion by the probe trial was substantially lower early in training (Supplementary Figure $\left.6 \mathrm{~B}, \chi^{2}(3, \mathrm{~N}=251)=15.9, \mathrm{p}=0.0012\right)$ and there was also increased attrition among participants assigned to late probes (Supplementary Figure $6 \mathrm{C}, \chi^{2}(3, \mathrm{~N}$ $=323)=8.5, \mathrm{p}=0.037)$. These patterns made assessment of shifts from place- to responsestrategies with extended training difficult to assess, compared to Experiment 1 and the pilot for Experiment 3, which used within-subject comparisons. 


\section{Transfer}

Probe trial choices were strongly predictive of success during strategy training (place or response) imposed after dual-solution training. Examining participants who completed at least four blocks of strategy training (probe trials no later than trial 80), participants who made placechoices and were transferred to place-training $(27 / 28,96 \%)$ reached criterion performance $(\geq$ $60 \%$ accuracy) in the first block of strategy-training, compared to $11 / 33(33 \%)$ transferred to response-training. For participants who made response-choices a similar pattern was observed (42/43, $98 \%$ of those transferred to response-training, compared to $5 / 32,16 \%$ of those transferred to place-training). While there was some improvement for participants assigned to the alternate strategy (e.g. place-choice assigned to response-training), by the fourth block of strategy-training only 14/27 (52\%) of participants who made a place-choice reached criterion for response-training, as did 13/24 (54\%) of participants who made a response-choice and were transferred to place-training.

For participants who made probe-choices adjacent to the place- or response-strategy defined locations $\left(\mid\right.$ error $_{\text {place }} \mid=1$ or $\mid$ error $\left._{\text {response }} \mid=1\right)$, accuracies were low in the first block of strategy training both for those who made place adjacent choices $(15 / 32,47 \%$, reached criterion in place-training, and 5/11, 45\%, reached criterion in response-training) and those who made response-location adjacent choices $(2 / 9,22 \%$, reached criterion for response-training, and 5/31, $16 \%$, for place-training). By the fourth block of place-training, participants who made placeadjacent choices improved to 21/24 (88\%) reaching criterion, compared to 5/9 (56\%) for response-adjacent choices. Less improvement was seen for response-training, where 5/9 (56\%) of participants who made a response-adjacent probe choice performed at criterion in the fourth block of training, and 7/26 (27\%) who made a place-adjacent probe choice. 


\section{Behavioral correlates of place- and response-learning}

Behavior during strategy training in Experiment 2 strongly discriminated between each imposed strategy. To replicate these findings, behavioral measures were examined during transfer to strategy training, comparing those who successfully acquired the strategy (accuracies $\geq 60 \%$ in the final block of strategy training) to those who did not. Separate ANOVAs were conducted for each measure with block as a within-subjects and with outcome (successful vs unsuccessful), strategy (place, response), and gender as between-subjects factors. The number of targets was initially included as a between-subjects factor, but there were no significant relationships to outcome (described in the Supplementary Materials). An initial analysis of dualsolution training found relationships to outcome (better performance during dual-solution training was associated with better transfer for place- and response-training, Figure $6 \mathrm{C}$ and Supplementary Figure 7A-C), and some interactions of gender with outcome and strategy. But, successful transfer to place- and response-training was not associated with specific patterns of behavior during dual-solution training (described in the Supplementary Materials).

During transfer (limited to participants who completed at least 6 blocks of strategy training), there were significant outcome $\times$ strategy interactions for route distance, excess rotation, and rotation reversals ( $\mathrm{ps}<0.001, \eta_{p}^{2}>0.12$, see Figure 6D and Supplementary Figure 7E-G). Post-hoc tests indicated that successful transfer to response-training was associated with significantly lower scores (more similar routes, less excess rotation, and fewer reversals), while successful transfer to place-training was associated with significantly higher scores on these measures. For pausing, there was also a significant gender $\times$ outcome $\times$ strategy interaction $(\mathrm{F}(1$, $\left.157)=5.1, \mathrm{p}=0.025, \eta_{p}^{2}=0.031\right)$, where post-hocs indicated that among females, success 
during response training was associated with a lower likelihood of pausing, while males showed less of a difference. Additionally, there was a main effect of block for distance overshoot ( $\mathrm{F}(5$, $\left.740)=2.8, p=0.017, \eta_{p}^{2}=0.018\right)$, but no other main effect or interaction for this measure (Supplementary Figure 7D) were significant ( $\left.\mathrm{ps}>0.09, \eta_{p}^{2} \mathrm{~s}<0.019\right)$.

\section{Discussion}

Across three experiments there were strong behavioral differences associated with the use of place- and response-strategies (on probe trials and during strategy training). Responsestrategies were associated with rapidly initiated, stereotyped trajectories, while place-strategies were associated with VTE-like behaviors (pausing and/or reorienting before making their choice), greater variability, and less efficient (i.e. direct) navigation. VTE-like reorientations were more likely to occur at high-cost decision points during the use of place-strategies, and resemble rodent vicarious trial-and-error (VTE) behavior, where animals hesitate, and make one or more orientations towards potential choices before committing to a decision (Muenzinger, 1938; Tolman, 1948). These results are consistent with the proposal that place-strategies generally represent model-based behavior, and that response-strategies generally represent model-free behavior.

VTE has emerged as a key index of deliberation in rodents (reviewed by Redish, 2016), and during navigation rats show increased VTE under conditions that are hippocampallydependent, or involve conflict between place- and response-strategies. VTE is commonly observed before rats have learned the correct decision, such as the direction to turn at a high-cost choice point in a maze (van der Meer, M. A. et al., 2010). During VTE in spatial tasks, hippocampal ensembles represent locations ahead of the animal's position (Johnson \& Redish, 
2007), while ventral striatal neurons represent reward (van der Meer, M. A. \& Redish, 2009), suggesting processing associated with planning and the evaluation of potential paths to goal locations.

While VTE is an important index of deliberation in rodents, less evidence exists in humans for a similar relationship between VTE, defined by changes in orientation between potential options, and deliberation or hippocampal-specific processing. However, eye movement patterns during visual search and discrimination have been proposed to share similarities with rodent VTE. Revisitations (return of fixation to a previously sampled item) are associated with better subsequent memory in humans, and are reduced in hippocampal amnesiacs (Voss et al., 2011), and are associated with improved performance and hippocampal activity during difficult perceptual discriminations (Voss \& Cohen, 2017). Additionally, in virtual navigation tasks, the results of Santos-Pata and Verschure (2018) suggest that behaviors analogous to rodent VTE can be identified using measures derived from those developed for rodent studies (Steiner \& Redish, 2014; Sweis et al., 2018). Similarly, the measures of VTE used in the present study were developed for a translational version of a rodent foraging task (Huynh et al., 2020) which found that the relationships between VTE and decision-making replicated results from rodents (Steiner \& Redish, 2014; Sweis et al., 2018), supporting VTE as a cross-species index of deliberation.

Together, these data are consistent with previous reports that rats and humans show rapid improvements in accuracy and slower development of a stable route (Schmitzer-Torbert, \& Redish, 2002; Schmitzer-Torbert, 2007), and that humans who use place-strategies on probe trials take longer, more circuitous paths to their goal, while in response-training females adopted more stable routes on average during response-training (Schmitzer-Torbert, 2007). These findings are also consistent with those of Santos-Pata and Verschure (2018) who demonstrated 
that during place-training in a multiple-T maze, VTE measures are enhanced at high-cost decision points and are associated with less efficient navigation (longer latencies to complete a trial). The present results extend these previous studies by demonstrating a specific relationship between response-strategies and the development of stereotyped, optimized behavior, and between place-strategies and VTE.

Place- and response-strategies depend on distinct set of brain networks, with place strategies requiring the function of the hippocampus and dorsomedial striatum, while responsestrategies require the dorsolateral striatum (Packard \& McGaugh, 1992; Packard \& McGaugh, 1996; Yin \& Knowlton, 2006). Rather than differing in the type of information used to guide behavior (e.g. spatial location for place-strategies, stimulus-response associations for responsestrategies), the results of navigation studies are consistent with the theory that the hippocampus and dorsomedial striatum support model-based behavior, while the dorsolateral striatum supports model-free behavior (Khamassi \& Humphries, 2012; Redish, 2013; Vikbladh et al., 2019). This is consistent with work demonstrating that spatial memory and model-based learning are correlated in humans, and the relationship is disrupted after hippocampal lesions (Vikbladh et al., 2019), and with the behavioral results of the present study, where place-strategies were associated with VTE-like behaviors, longer latencies and more variable behavior, while response-strategies were associated with rapid, stereotyped (low-variability) behaviors which develop slowly across experience.

During dual-solution training in a multiple-T maze, participants tend to shift to the use response-strategies, from the use of place-strategies early to response strategies late in training (Schmitzer-Torbert, 2007, and Experiment 1 of the present report), or from a choice that did not indicate the clear use of a strategy early to a response-strategy late in training (Experiment 3 
pilot). These results are consistent with work showing that in rats trained under dual-solution conditions, place-strategies are more common early in training, and response strategies become more common later in training (Packard \& McGaugh, 1996), and from humans tested in a radial maze task (Iaria, Petrides, Dagher, Pike, \& Bohbot, 2003). Participants who make responsechoices on the probe trial behave overall as if they have not detected a change in their starting location: their reaction times and (egocentric) trajectories are very similar to those used during dual-solution training, and they take efficient, direct paths to their goal. However, it is not the case that a response-choice simply indicates a failure of self-localization on the first novel experience (failing to detect that one is in a new location on the probe trial), as a substantial proportion of participants who make response-choices on probe trials continue to be impaired when a place-strategy is imposed after the probe (in Experiment 3 and the pilot).

Indeed, probe trial choices were strongly predictive of successful transfer to the imposed strategy in Experiment 3 (and the pilot study), indicating that the strategy revealed on the probe trial did reflect the navigation strategy that participants would most easily express during strategy training. In contrast, in Experiment 2, success in acquiring one strategy (place or response) was unrelated to success on the alternate strategy (learned for a separate target), so it appears reasonable to hypothesize that participants who successfully transferred to the imposed strategy (e.g. place) would not have been strongly impaired if the alternate strategy (response in this case) had been imposed from the outset. The data from these experiments indicate that the strategy observed by participants on the probe trial is a reliable index of the navigation strategy used by the participants to reach a goal that was learned under dual-solution conditions.

However, behavioral measures during dual-solution training were not predictive of probe trial behavior, contrary to a previous report (Schmitzer-Torbert, 2007), though participants who 
made place-choices did tend to show elevated rotation reversals and pausing in the subsequent dual-solution trial after the probe. This result appears similar to results with rats trained to switch between place- and response-strategies on a plus maze (Schmidt et al., 2013), where VTE was elevated on trials where rats made errors (regardless of strategy), and on the subsequent trial. In that study, the authors proposed that the omission of reward on an error trial may have increased the use of a deliberative strategy on the following trial, reflected in the increased VTE. In the present study, it may be that participants who expressed a place-strategy on the probe (and were rewarded for doing so) were then biased to continue the deliberative strategy on the following trial.

While I predicted that dual-solution would predict probe trial behavior and transfer to strategy training, there is evidence in humans that hippocampal and striatal contributions to navigation develop in parallel, rather than competitively (Simon \& Daw, 2011), and it is possible that during dual-solution conditions, both hippocampal and striatal systems guide behavior. Under conflict (probe trials, strategy training), areas such as the ventromedial prefrontal cortex could be activated if both the hippocampal and striatal systems are active (Simon \& Daw, 2011) and mediate conflict between the hippocampal and striatal systems. Alternatively, the failure of dual-solution behavior to predict probe trial and strategy-training behavior may be an artifact of the virtual navigation tasks used, where participants learned to find the location of one or two targets in relatively simple environments. Consideration of a wider variety of navigation tasks would be beneficial, to determine if these behavioral measures can identify the navigation strategy that is in use, or transitions in strategy, during dual-solution conditions.

Together, the results of these studies support the identification of place-strategies in navigation as an example of model-based behavior, and response-strategies as an example of 
model-free behavior. Consistent with previous studies, VTE is enhanced during the expression of place-strategies, while stereotyped behaviors are observed during the expression of responsestrategies. The results also support the use of VTE as a cross-species index of deliberation, and may allow for the behavioral identification of episodes of deliberation during human virtual navigation, which may allow for the study of neural processing related to the use of search-andevaluation behaviors (similar to studies in rodents).

\section{Materials and Methods}

\section{Experiment 1}

\section{Participants}

A total of 73 male undergraduate students (mean age $=19.3$, range $=17$ to 22 ) participated in the study. Four subjects did not complete the experiment, and were excluded from analyses. Students were recruited from undergraduate psychology courses at Wabash College, in the context of undergraduate research projects on the effect of glucose administration and working memory load on spatial navigation. Neither the glucose manipulation $(n=20)$, nor the working memory manipulation $(\mathrm{n}=49)$ were found to impact learning on these tasks, and so are not described further. Participants either completed the study independently $(n=49)$, using a personal computer or a computer in campus computer lab, or were tested in-person $(n=20)$ for the glucose manipulation. Participants were offered extra credit for participation and additionally motivated by monetary prizes awarded to the participants with the fastest trial time (for any trial of the experiment) and the fastest average trial time (across all trials in the experiment). Participants provided informed consent, and all procedures were approved by Wabash College's Human Research Committee. 


\section{Task}

Participants were trained in virtual environments (see Figure 1 for examples) created using commercially available software (Game Maker 6.1, Mark Overmars, http://www.yoyogames.com/gamemaker). In each experiment, movement through the virtual environments was controlled by using the arrow keys on the computer keyboard (the up and down arrow keys moved the participant forward and backward, respectively, and the left/right arrow keys were used to turn). A $360^{\circ}$ image of a sunset was used to provide distal cues (www.1000skies.com, see Supplementary Figure 1A). Textures for other game objects were obtained and used by permission of David Gurrea (http://www.davegh.com/index.php/en/floor).

Training consisted of pre-training in a virtual Barnes maze (all participants) and placetraining in the multiple-T maze $(n=41$ participants, 20 tested in-person). In the Barnes maze, participants were placed in the middle of a grass field, and 20 cylinders were placed around them in a circle. For each participant, one of these cylinders was rewarded, and participants were required to find the correct cylinder as quickly as possible during 18 trials of training. To find the correct cylinder, participants were required to move close to the cylinder, causing it to drop to the ground and reveal if their target was inside. If not, they continued to select additional cylinders until they found the target object.

The multiple-T maze was designed to be symmetrical around $180^{\circ}$ rotations, and to contain two starting locations (See Figure 1B-C). Each starting location led, via three turns, to eight possible goal locations. A cylinder, like those used in the Barnes maze, were placed at the end of each goal arm, giving a total of eight cylinders. 
In the main phase of training, participants completed 60 training trials from one starting location, searching for a target placed in one of the goal cylinders. In one sample $(n=28$, tested independently), participants also searched for a secondary target on some trials (60 total), starting from the same location (and thus had 120 trials of total training, with the trials for the two targets pseudorandomly interspersed). For the principal target, two probe trials were also administered after the $10^{\text {th }}$ and $55^{\text {th }}$ training trial. On probe trials, participants were placed in the opposite starting arm from that used on normal trials. To make the probe trial less obtrusive, whichever goal cylinder was chosen first was designated as correct, and the target object was moved to that location before the cylinder dropped away.

\section{Analyses}

Analyses were conducted in Matlab for all experiments. ANOVAs were conducted using the simple_mixed_anova.m function (Caplette, 2017). Significant main effects and interactions were analyzed using Tukey HDS post-hoc tests $(\alpha=0.05)$. For within-subjects factors involving more than two levels, the Greenhouse-Geisser correction is reported (as pcorrected) for main effect or interactions where there was a significant violation of sphericity and the corrected p-value exceeded $\alpha=0.05$.

Blocks. Analyses of task acquisition were performed after averaging dependent measures in blocks. Six blocks of three trials each were used for the Barnes maze, six blocks of four trials each were used for multiple-T pre-training, and twelve blocks of five trials each were used for multiple-T training for the primary target. 


\section{Behavioral measures}

Accuracy. For multiple-T maze training, each trial was scored as either correct (= to 100$)$ or incorrect $(=0)$ based on the subject's first choice.

Pre-training. For pre-training on the Barnes maze, an error score was also calculated for the participant's first choice, and this error indicated how far (in terms of cylinders) the participant's first choice was from the correct cylinder. Perfect performance was indicated by error scores of zero.

Route distance. The similarity between trajectories was measured for each pairs of trials $i$ and $j$ was using route distance, a measure of the average distance between position samples, aligned to the start of each trial (Schmitzer-Torbert, 2007). Trials were truncated in each pair to the shorter of the two trials. Route distance was calculated for trials $i$ and $j$ as

$$
\text { Route distance }=\frac{\sum_{k=1}^{n} \sqrt{\left(x_{i}(k)-x_{j}(k)\right)^{2}+\left(y_{i}(k)-y_{j}(k)\right)^{2}}}{n}
$$

Where $x(k)$ and $y(k)$ are the position of the participant at sample $k$, relative to the start of the trial, and $n$ is the number of position samples in the shorter of the two trials (truncated at the time when the participant made their first choice). Route distance approaches 0 in cases where the trajectory taken through the maze (position as a function of time) on both trials is identical. Route distance was only defined for pairs of trials in which the egocentric location (relative to the starting location) of the participant's first choice was the same (comparison of trials where participants selected two different egocentric targets would be large by definition). A single value for route distance was assigned to each trial $i$ (where the participant made an egocentric choice $r$ ) by averaging the $\log _{10}$ transformed route distance scores between trial $i$ and last five trials in which egocentric choice $r$ was made (the final trajectory adopted for $r$ ). 
Distance and latency scores. On each trial, the latency and distance travelled up to when participants made their first choice was compared to the minimum achieved across all participants for the same (egocentric) choice $(r)$. The ratio of the latency/distance achieved to the minimum achieved across the sample was defined as the distance/latency score, with a score of 1 being equal to the shortest/fastest trial for the location chosen.

VTE behaviors. In the starting location (the first decision point), several behavioral measures of vicarious trial-and-error were assessed beyond dwell time. These included the total distance travelled ( $\log _{10}$ units), total rotation (sum of the absolute value of changes in rotation), number of reversals of rotation direction, and pausing ( 1 = paused at least once for a minimum of $500 \mathrm{~ms}$ before exiting).

Probe trials. On probe trials, subjects were classified as using a place-strategy if they chose the same cylinder that had been rewarded on normal training trials, and they were classified as using a response-strategy if they chose the cylinder that was located in the same (egocentric) position relative to their new starting location. All other choices were classified as "other" and were not thought to represent the effective use of either a place- or response-strategy.

\section{Experiment 2}

\section{Participants}

Undergraduate participants were recruited from a small liberal-arts college in Illinois and from a large research university in Minnesota. A total of 104 participants completed the virtual navigation task, of whom 102 submitted gender information. Of the 102 participants who completed the task, 39 were submitted from Knox College (18-22 years old, mean age $=19.7$ years $[\mathrm{SD}=1.1], 24$ females), and 47 from University of Minnesota (18-38 years old, mean age 
$=20.3[\mathrm{SD}=3.6], 31$ females ), and 16 from Wabash College (all males, age was not collected for the Wabash sample, but the student body is primarily aged 18-22). Participants were compensated with extra credit or course credit for their participation. The research study was approved by the Institutional Research Board of each institution. Participants were tested in public computer labs (using laptops or desktop computers) or in smaller research labs.

\section{Task}

Participants were trained to navigate a circular island, surrounded by water on all sides in a virtual environment (Figure 3A-C) created using the Torque Game Engine (Garage Games, http://www.garagegames.com). Trees surrounded the edge of the island, and mountains were visible in the distance. The island was covered with an irregular grid of large rocks which appeared to be placed at random, which were symmetrical around every $90^{\circ}$ rotation. Participants started each trial in one of four locations, and searched for targets placed in one of four cylinders located on the island and were not visible from the starting locations. After incorrect choices, the selected cylinder was raised up, and could then be seen from other locations on the island.

Training consisted of pre-training, followed by place- and response-training (with the order counterbalanced across participants). In each phase, participants searched for a different item (spheres of different colors) indicated by an onscreen icon, and the change in the identity of the target was the only signal that the participant had advanced to a new phase of training. In pretraining, participants started once from each the four starting locations, and the target was placed at one of the four goal locations, and was visible before the participant arrived at the cylinder. During pre-training, neither place- nor response-strategies were rewarded. During place-training, the location of the target was always in the same (allocentric) location on the virtual island, while 
during response-training, the location of the target was fixed relative (egocentric) to the participant's starting location.

\section{Measures}

Behavior was assessed on each trial up to the first choice. Measures used from Experiment 1 included route distance, distance overshoot, total rotation, rotation reversals, and pausing. Reaction time (milliseconds) from the start of the trial until participants began moving, and excess rotation, the difference between the total angular rotation and the minimum amount of rotation needed to turn to the selected target location were also measured. For analysis, dependent measures were averaged in four blocks of 5 trials for each strategy.

\section{Experiment 3}

\section{Participants}

Undergraduate participants were recruited from two small liberal-arts colleges (Wabash College and DePauw University in Indiana and Knox College in Illinois), and online through email advertisement, posting on Craigslist and internet psychology research boards, and through Amazon's Mechanical Turk Service. Partial sessions were submitted from 251 participants (220 of whom submitted their gender), and complete sessions (for which at least 99 trials were submitted) were received from 207 participants (80 females, 18-59 years old, mean age $=25.4$ years $[\mathrm{SD}=8.9]$, of which 76 were undergraduates [27 females]). Undergraduates were compensated with extra credit, course credit or a gift card for their participation. The majority of the non-undergraduate participants were recruited through mTurk (restricted to workers with an approval rate for mTurk assignments of $95 \%$ or greater, and no location restrictions were used) 
and were paid \$5.25 USD. For the non-undergraduate participants $(n=131)$, most were estimated to likely have participated from the United States $(n=86,44$ females) based on their IP address. The research study was approved by the Institutional Review Board of Wabash College. Undergraduates were tested in public computer labs (using laptops or desktop computers) and other participants used their personal computers.

\section{Task}

Participants were tested in a virtual environment consisting of a Barnes maze placed on a circular platform surrounded by water and mountain landscape (Figure 5A-B). The task was implemented using the Unity3D game engine (version 2, www.unity3d.com) and embedded in a webpage. Participants began each trial from one of four starting locations near the center of the island, surrounded by eight cylinders which represented potential locations for hidden targets. Participants completed a total of 100 trials.

Training began with dual-solution training (using fixed starting locations), where participants were instructed to find a target on each trial hidden in one of the cylinders. Participants were then switched to place- or response-training after 10-90 dual-solution trials. The first trial of strategy-training (randomly selected between trials 11-91), was considered a probe trial, and participants were placed in the starting position opposite that used in the dualsolution trials (rotated $180^{\circ}$ relative to the previous trial). Starting with the probe trial, participants were transferred to strategy-training, where participants began each trial from one of the four starting locations and a place- or response-strategy was imposed for the remainder of the session. 


\section{Behavioral measures}

On each trial, behaviors up to the time participants made their first choice was analyzed. Task acquisition during dual-solution and strategy-training was assessed by accuracy (as in the earlier experiments). Measures used from Experiments 1 and 2 included route distance, distance and latency overshoot, excess rotation, total rotation, rotation reversals, and pausing. As trajectories were observed to become more variable under some conditions (transitioning from dual-solution to place-training), a local measure of route distance was used for each trial, defined for trial $i$ (where $r$ is defined as the egocentric location chosen on trial $i$ ) as the average $\log _{10}$ transformed route distance between $i$ and the last use of $r$ before $i$ (in trials 1 to $(i-1)$ ) and next use of $r$ after $i$ (in trials $(i+1)$ to $n$ ).

Reaction time data were not analyzed, as accurate reaction times were missing from a subset of trials due to an error in marking the start of each trial. For analysis, dependent measures were averaged in blocks of 5 trials, aligned to the start of dual-solution training or to the probe trial (to analyze transfer).

Probe trials. As in the previous experiments, participants were classified as using a place-strategy, response-strategy, or making an "other" choice on the probe trial. Analyses were also conducted on probe choices classified as "place-adjacent" or "response-adjacent" if participant's choice was adjacent to the location defined by the place- or response-strategy.

\section{Acknowledgements}

I would like to thank Angus MacDonald III, who assisted with the experimental design for Experiment 2, and whose lab collected the data presented for that study, and Barron Hewetson, who collected the data presented in Experiment 1. 


\section{References}

Bett, D., Allison, E., Murdoch, L. H., Kaefer, K., Wood, E. R., \& Dudchenko, P. A. (2012). The neural substrates of deliberative decision making: Contrasting effects of hippocampus lesions on performance and vicarious trial-and-error behavior in a spatial memory task and a visual discrimination task. Frontiers in Behavioral Neuroscience, 6, 70. doi:10.3389/fnbeh.2012.00070 [doi]

Caplette, L. (2017). Simple mixed anova in MATLAB. https://www.mathworks.com/matlabcentral/fileexchange/64980-simple-mixed-anova-for$\underline{\text { any-design }}$

Doeller, C. F., King, J. A., \& Burgess, N. (2008). Parallel striatal and hippocampal systems for landmarks and boundaries in spatial memory. Proceedings of the National Academy of Sciences of the United States of America, 105(15), 5915-5920. Retrieved from https://wabash.on.worldcat.org/oclc/5551727960

Huynh, T., Alstatt, K., Abram, S. V., \& Schmitzer-Torbert, N. C. (2020). Vicarious trial-anderror is enhanced during deliberation in human virtual navigation in a translational neuroeconomic task. doi:https://doi.org/10.1101/2020.02.21.954230

Iaria, G., Petrides, M., Dagher, A., Pike, B., \& Bohbot, V. D. (2003). Cognitive strategies dependent on the hippocampus and caudate nucleus in human navigation: Variability and change with practice. Journal of Neuroscience, 23(13), 5945. 
Johnson, A., \& Redish, A. D. (2007). Neural ensembles in CA3 transiently encode paths forward of the animal at a decision point. Journal of Neuroscience, 27(45), 12176-12189. doi:10.1523/JNEUROSCI.3761-07.2007

Khamassi, M., \& Humphries, M. D. (2012). Integrating cortico-limbic-basal ganglia architectures for learning model-based and model-free navigation strategies. Frontiers in Behavioral Neuroscience, 6, 79. doi:10.3389/fnbeh.2012.00079 [doi]

Muenzinger, K. F. (1938). Vicarious trial and error at a point of choice: I A general survey of its relation to learning efficiency Clark University. doi:10.1080/08856559.1938.10533799

Packard, M. G., \& McGaugh, J. L. (1992). Double dissociation of fornix and caudate nucleus lesions on acquisition of two water maze tasks: Further evidence for multiple memory systems. Behavioral Neuroscience, 106(3), 439-446. doi:10.1037/0735-7044.106.3.439

Packard, M. G., \& McGaugh, J. L. (1996). Inactivation of hippocampus or caudate nucleus with lidocaine differentially affects expression of place and response learning. Neurobiology of Learning and Memory, 65(1), 65-72. doi:10.1006/nlme.1996.0007

Redish, A. D. (2016). Vicarious trial and error. Nature Reviews.Neuroscience, 17(3), 147-59. doi:10.1038/nrn.2015.30

Redish, A. D. (2013). The mind within the brain: How we make decisions and how those decisions go wrong. New York, NY: Oxford University Press. Retrieved from https://login.wcproxy.palni.edu/login?url=http://search.ebscohost.com/login.aspx?direct=tru $\mathrm{e} \& \mathrm{db}=\mathrm{psyh} \& \mathrm{AN}=2013-24085-000 \&$ site $=$ ehost-live $\&$ scope $=$ site 
Santos-Pata, D., \& Verschure, P. (2018). Human vicarious trial and error is predictive of spatial navigation performance. Frontiers in Behavioral Neuroscience, 12

Schmidt, B., Papale, A., Redish, A. D., \& Markus, E. J. (2013). Conflict between place and response navigation strategies: Effects on vicarious trial and error (VTE) behaviors. Learning \& Memory, 20(3), 130-138. doi:10.1101/lm.028753.112

Schmitzer-Torbert, N. C. (2007). Place and response learning in human virtual navigation: Behavioral measures and gender differences. Behavioral Neuroscience, 121(2), 277-290. doi:10.1037/0735-7044.121.2.277

Schmitzer-Torbert, N., \& Redish, A. D. (2002). Development of path stereotypy in a single day in rats on a multiple-T maze. Archives Italiennes De Biologie, 140(4), 295-301.

Schmitzer-Torbert, N., \& Redish, A. D. (2004). Neuronal activity in the rodent dorsal striatum in sequential navigation: Separation of spatial and reward responses on the multiple T task. Journal of Neurophysiology, 91(5), 2259-2272. doi:10.1152/jn.00687.2003 [doi]

Simon, D. A., \& Daw, N. D. (2011). Neural correlates of forward planning in a spatial decision task in humans. The Journal of Neuroscience : The Official Journal of the Society for Neuroscience, 31(14), 5526-39. doi:10.1523/JNEUROSCI.4647-10.2011

Steiner, A. P., \& Redish, A. D. (2014). Behavioral and neurophysiological correlates of regret in rat decision-making on a neuroeconomic task. Nature Neuroscience, 17(7), 995-1002. doi:10.1038/nn.3740 
Sweis, B. M., Abram, S. V., Schmidt, B. J., Seeland, K. D., MacDonald III, A. W., Thomas, M. J., \& Redish, A. D. (2018). Sensitivity to "sunk costs" in mice, rats, and humans. Science (New York, N.Y.), 361(6398), 178-181. doi:10.1126/science.aar8644

Tolman, E. C. (1948). Cognitive maps in rats and men American Psychological Association. doi:10.1037/h0061626

van der Meer, M. A., Johnson, A., Schmitzer-Torbert, N. C., \& Redish, A. D. (2010). Triple dissociation of information processing in dorsal striatum, ventral striatum, and hippocampus on a learned spatial decision task. Neuron-Cambridge Ma-, 67(1), 25-32. Retrieved from https://wabash.on.worldcat.org/oclc/649786044

van der Meer, M. A., \& Redish, A. D. (2009). Covert expectation-of-reward in rat ventral striatum at decision points. Frontiers in Integrative Neuroscience, 3, 1. doi:10.3389/neuro.07.001.2009

Vikbladh, O. M., Meager, M. R., King, J., Blackmon, K., Devinsky, O., Shohamy, D., ... Daw, N. D. (2019). Hippocampal contributions to model-based planning and spatial memory. Neuron, 102(3), 683-693. doi:10.1016/j.neuron.2019.02.014

Voss, J. L., \& Cohen, N. J. (2017). Hippocampal-cortical contributions to strategic exploration during perceptual discrimination. Hippocampus, 27(6), 642-652.

Voss, J. L., Warren, D. E., Gonsalves, B. D., Federmeier, K. D., Tranel, D., \& Cohen, N. J. (2011). Spontaneous revisitation during visual exploration as a link among strategic 
behavior, learning, and the hippocampus. Proceedings of the National Academy of Sciences of the United States of America, 108(31), 12581-12582.

Yin, H. H., \& Knowlton, B. J. (2006). The role of the basal ganglia in habit formation. Nature Reviews Neuroscience, 7(6), 464-476. 